\title{
A mutant in one of two exbD loci of a TonB system in Flavobacterium psychrophilum shows attenuated virulence and confers protection against cold water disease \\ Correspondence \\ José A. Guijarro \\ jaga@fq.uniovi.es \\ Received 21 July 2007 \\ Revised 2 January 2008 \\ Accepted 4 January 2008 \\ Beatriz Álvarez, Javier Álvarez, Aurora Menéndez and José A. Guijarro \\ Área de Microbiología, Departamento de Biología Funcional, Facultad de Medicina, IUBA, Universidad de Oviedo, 33006 Oviedo, Spain

\begin{abstract}
Flavobacterium psychrophilum is a psychrotrophic fish-pathogenic bacterium that causes cold water disease (CWD) in salmonids. By means of Tn4351 mutagenesis a mutant named FP1033, deficient in growth on iron-depleted medium, was previously isolated. FP1033 recovered the parental phenotype in the presence of iron. The gene disrupted by the transposon in this mutant encoded a protein with similarity to ExbD proteins, which are members of the TonB complex system involved in iron uptake mediated by siderophores. Analysis of the DNA surrounding the transposon insertion showed the presence of a ton $B$ cluster of genes composed of exbB, two exbD (exbD1 and exbD2) and tonB loci. RT-PCR analysis and complementation studies indicated that these genes are transcribed as an operon and that the exbD2::Tn4351 phenotype was caused by the lack of ExbD2. FP1033 showed decreased virulence and conferred a high level of protection in rainbow trout fry after vaccination. This is believed to be the first report of a

F. psychrophilum attenuated strain that induces a protective immune response in rainbow trout against CWD. These results suggest that the exbD2 locus from this particular TonB system is a suitable target to generate a live attenuated vaccine.
\end{abstract}

\section{INTRODUCTION}

One of the most important pathogens of salmonids is Flavobacterium psychrophilum. This Gram-negative, psychrotrophic and 'fastidious' bacterium belongs to the CytophagaFlavobacterium-Bacteroides group. F. psychrophilum is the aetiological agent of the cold water disease (CWD) that occurs at temperatures below $12{ }^{\circ} \mathrm{C}$ and has considerable economic significant to aquaculture producers (for a review see Nematollahi et al., 2003). The control of outbreaks is currently based on fish management strategies and the use of antimicrobial therapy. However, these treatments are costly, antibiotic-resistant bacteria can arise (Bruun et al., 2000; Schmidt et al., 2000; Izumi \& Aranishi, 2004) and, additionally, the health of human and terrestrial animals could be affected in different ways by this use of antibiotics (Cabello, 2006). An available commercially licensed vaccine has not been developed up to now. Nevertheless, several studies testing vaccination with fractions or inactivated whole cells have been done. It has been described that whole formalin-killed cells (Kondo et al., 2003), a

Abbreviations: CWD, cold water disease; RPS, relative percentage survival.

The GenBank/EMBL/DDBJ accession number for the tonB operon and surrounding DNA sequence is EF408823.
Sarkosyl-insoluble membrane fraction (Rahman et al., 2002) and different molecular mass fractions (LaFrentz et al., 2004) of $F$. psychrophilum confer protection against the disease. A recent study showed that membrane vesicles contained in the stationary-phase culture supernatant had an adjuvant effect (Aoki et al., 2007). The authors of that study suggested that a combination of membrane vesicles and cells is necessary to obtain efficient protection. Additionally, an $\mathrm{OmpH}$-like surface protein named P18 can induce a protective immune response in fish (Dumetz et al., 2006).

In recent years advances in cultivation (Michel et al., 1999), diagnosis (del Cerro et al., 2002), experimental infections (Garcia et al., 2000) and genetic techniques for DNA transfer (Álvarez et al., 2004) have been reported for F. psychrophilum. However, knowledge on the mechanism of pathogenesis is still limited. Iron is an essential element for the majority of bacterial life, and therefore microorganisms have developed multiple iron-acquisition systems (Wyckoff et al., 2007). Due to the reduced availability of iron in host tissues, the ability to obtain iron in vivo by micro-organisms is considered an important factor in pathogenicity (Payne, 1993; Wooldridge \& Williams, 1993; Braun, 1995). For Gram-negative bacteria, many of the iron-acquisition systems involve the presence of different kinds of outer-membrane receptors that bind a specific 
ligand (iron-siderophore complex, haemin, haemoglobin, transferrin or lactoferrin) (Andrews et al., 2003). These iron compounds are then introduced into the cell by means of the TonB system, which is an energy-transduction complex required for all high-affinity iron-transport systems. It is composed of TonB, ExbB and ExbD, found in the cytoplasmic membrane of many Gram-negative bacteria, where they energize iron uptake (for review see Braun \& Braun, 2002; Andrews et al., 2003; Wandersman \& Delepelaire, 2004). In pathogenic bacteria, these ironacquisition systems allow the bacteria to scavenge iron and grow inside the host. Therefore, the TonB system is associated with mechanisms involved in the progress of infection in several important pathogens such as Vibrio cholerae (Seliger et al., 2001), Shigella dysenteriae (Reeves et al., 2000; Payne et al., 2006), uropathogenic Escherichia coli (Torres et al., 2001) and Bordetella pertussis (Pradel et al., 2000). In the bacteria fish pathogens Yersinia ruckeri (Fernández et al., 2004) and Vibrio anguillarum (Stork et al., 2004) a relationship between genes related to TonB systems and virulence has been reported. In the case of F. psychrophilum the production of siderophore-like molecules was indicated in a study by Møller et al. (2005).

In an attempt to discover more about the pathogenesis mechanisms of F. psychrophilum, a set of mutants deficient in growth in iron-limited conditions were previously isolated using a Tn4351-mutagenesis system (Álvarez et al., 2006). In the present work, one of these mutants, designated FP1033, was further analysed. This mutant had a transposon insertion within a gene with identity to the exbD locus from a TonB system. Sequence, complementation and physiological studies defined a TonB system in F. psychrophilum. Interestingly, this system included two exbD (exbD1 and exbD2) homologous loci. $\mathrm{LD}_{50}$ experiments revealed that FP1033 had reduced virulence, indicating that the exbD2 gene plays an important role in virulence of $F$. psychrophilum. Furthermore, FP1033, as an attenuated strain, was capable of inducing a protective immune response in rainbow trout fry a few weeks post-vaccination. These results open the possibility of using an attenuated live vaccine against CWD.

\section{METHODS}

Bacterial strains, plasmids and growth conditions. E. coli strain S17-12pir (Simon et al., 1983), used to transfer DNA into $F$. psychrophilum, was grown at $37^{\circ} \mathrm{C}$ in $2 \times \mathrm{TY}$ medium (10 g tryptone,

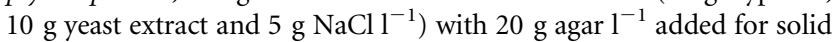
medium. F. psychrophilum strain THC02-90 was grown at $20{ }^{\circ} \mathrm{C}$ on solid EAOS medium (Michel et al., 1999) or at $12{ }^{\circ} \mathrm{C}$ and $18{ }^{\circ} \mathrm{C}$ in nutrient broth (NB; Pronadisa), as previously described (Álvarez et al., 2004). To evaluate growth on iron-depleted conditions, the iron chelator 2,2' -dipyridyl was added to NA (nutrient agar, consisting of $\mathrm{NB}$ with $1.5 \%, \mathrm{w} / \mathrm{v}$, agar) or NB at a final concentration of $50 \mu \mathrm{M}$. For selective growth, antibiotics were added when needed at the following concentrations: $10 \mu \mathrm{g}$ erythromycin $\mathrm{ml}^{-1}$ (to maintain the Tn4351 in the mutant); $100 \mu \mathrm{g}$ ampicillin $\mathrm{ml}^{-1}$; and $10 \mu \mathrm{g}$ tetracycline $\mathrm{ml}^{-1}$. The plasmids and primers used are listed in Table 1.
Identification of insertion site of Tn4351 in FP1033 and amplification of the DNA surrounding $F$. psychrophilum exbD2 by inverse PCR. Tn 4351 was introduced into F. psychrophilum THC02-90 by conjugation, and selection of mutants with defects in motility, proteolytic activity or the ability to grow in the presence of 2,2'-dipyridyl was done as previously described (Álvarez et al., 2006). The site of Tn 4351 insertion and the surrounding DNA in FP1033 was analysed by Southern blot as described by Álvarez et al. (2006).

To perform inverse PCR, digestion of chromosomal DNA of the mutant with HindIII followed by religation was carried out. The resulting circular molecules were used as template to amplify the sequences adjacent to the $\operatorname{Tn} 4351$ insertion site using the $\mathrm{Tn} 4351$-specific pair of primers TN-1/IS4351-F (Table 1) and the Certamp long amplification kit (BIOTOOLS B\&M Laboratories). DNA surrounding exbD2 was amplified from DNA of the wild-type strain THC02-90 previously digested with $\mathrm{Xba \textrm {I }}$ and religated. The following pairs of primers were used: INV-A/INV-B, INV-A/INV-C, INV-D/INV-E and INV-F/INV-G (Table 1).

Nucleic acid sequencing. Automated fluorescence sequencing was performed at the Oviedo University DNA analysis facility using BigDye 3.1 Terminator chemistry on an ABI PRISM 3100 Genetic Analyzer platform (Applied Biosystems). Sequences were compared to databases by using BLAST from the National Center for Biotechnology Information (NCBI) website (http://www.ncbi.nlm.nih.gov/BLAST/).

RT-PCR. Total RNA was obtained from $150 \mu$ l of late-exponentialphase cultures of the wild-type THC02-90 and the mutant FP1033 grown in NB. RNA was isolated using the RNeasy Mini kit (Qiagen) and treated twice with RNase-Free DNase (Promega) to eliminate traces of DNA. RT-PCRs were performed using Superscript One-Step RT-PCR with Platinum Taq (Invitrogen Life Technologies); 15 ng RNA was used in each reaction. PCR control reactions using GoTaq Flexi DNA polymerase (Promega) were performed to determine whether RNA was free of contaminant DNA. Primers used (RT-exbBF, RT-exbD1-R, RT-exbD2-R, RT-exbD2-F, RT-tonB-R, RT-tonB-F, RT-orf3-R, RT-Tn) are listed in Table 1.

Complementation of the exbD2::Tn4351 mutation. Different amplicons containing (i) exbD2, (ii) $\operatorname{exbD2}$ and $\operatorname{ton} B$, and (iii) $\operatorname{exbD2}$, tonB and orf2 were cloned in pCP23 in order to complement FP1033. The exbD2 gene was amplified by PCR using the Certamp long amplification kit (BIOTOOLS B\&M Laboratories) and primers ExbD2-F and ExbD2-R (Table 1). exbD2 and tonB were amplified using the primers ExbD2-F and TonB-R (Table 1), and a DNA fragment that contained $\operatorname{exbD2}$, tonB and orf2 was amplified using the primers ExbD2-F and Orf2-R (Table 1). BamHI and PstI restriction sites had been introduced in the sequences of the forward primers and reverse primers, respectively, to clone the obtained PCR products digested with BamHI and PstI into pCP23 that had been digested with the same restriction enzymes. The resulting plasmids were named $\mathrm{pJB} 1$, which contained $\operatorname{exbD2}$, pJB2, which contained $\operatorname{exbD2}$ and tonB, and $\mathrm{pJB} 3$, which contained $\operatorname{exbD2}$, tonB and orf2. For complementation analysis pJB1, pJB2 and pJB3 were first introduced into F. psychrophilum THC02-90 by conjugation. The plasmids were isolated from wild-type $F$. psychrophilum and transferred by electroporation into F. psychrophilum FP1033, as previously described (Álvarez et al., 2004).

Growth curve and production of siderophores by THC02-90 and FP1033. Flasks containing NB were inoculated with $1 / 300$ volume of stationary-phase cultures of THC02-90 and FP1033 and incubated at 250 r.p.m. and $12{ }^{\circ} \mathrm{C}$. Growth was determined by measuring $\mathrm{OD}_{525}$. To test the effect on growth of inorganic iron, $\mathrm{FeCl}_{3}$ was added to NB to a concentration of $20 \mu \mathrm{M}$. 
Table 1. Plasmids and primers used in this study

\begin{tabular}{|c|c|c|}
\hline Plasmid or primer & Description and/or sequence & Source or reference \\
\hline \multicolumn{3}{|l|}{ Plasmids* } \\
\hline pCP23 & ColE1 ori, (pCP1 ori), $\mathrm{Ap}^{\mathrm{r}}\left(\mathrm{Tc}^{\mathrm{r}}\right)$, E. coli-F. psychrophilum shuttle plasmid & Agarwal et al. (1997) \\
\hline pJB1 & pCP23 containing exbD2, $\mathrm{Ap}^{\mathrm{r}}\left(\mathrm{Tc}^{\mathrm{r}}\right)$ & This study \\
\hline pJB2 & pCP23 containing exbD2 and tonB, $\mathrm{Ap}^{\mathrm{r}}\left(\mathrm{Tc}^{\mathrm{r}}\right)$ & This study \\
\hline pJB3 & 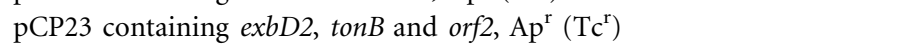 & This study \\
\hline \multicolumn{3}{|c|}{1} \\
\hline IS4351-F & $5^{\prime}$-GACTTGGATACCTCACGCC-3' & McBride et al. (2003) \\
\hline $\mathrm{TN}-1$ & 5'-GGACCTACCTCATAGACAA-3' & Álvarez et al. (2006) \\
\hline INV-A & 5'-CAAATCTACCATTGCAGTTA-3' & This study \\
\hline INV-B & 5'-GGTTACCGAATAGTCTTAGG-3' & This study \\
\hline INV-C & 5'-TTAGCGAAGTGGTAAGCATA-3' & This study \\
\hline INV-D & 5'-CAGATTCTCACAAATGGACGG-3' & This study \\
\hline INV-E & 5'-CCTGCAAGAGTTCCTAACGAC-3' & This study \\
\hline INV-F & 5'-TCCATTCCTTCAACTGA-3' & This study \\
\hline INV-G & 5'-CTTCCAATCATAGATGACC-3' & This study \\
\hline RT-exbB-F & $5^{\prime}$-CAGGTGGTGGTGGAGCAGA-3' & This study \\
\hline RT-exbD1-R & $5^{\prime}$-GGCACTACTCTTAGCAATTAGA-3' & This study \\
\hline RT-exbD2-F & 5'-CTGGCGACGGCGGTGGAA-3' & This study \\
\hline RT-exbD2-R & 5'-GATCTCCAGTTTCAGCAATTAC-3' & This study \\
\hline RT-tonB-F & 5'-GGTTTGCCAATGCTTCTCGATA-3' & This study \\
\hline RT-tonB-R & 5'-GTATTGTCTACAATCTCAGCG-3' & This study \\
\hline RT-orf3-R & 5'-CGATCCGTTGCAAGTGGAGT-3' & This study \\
\hline RT-Tn & 5'-GTTGCATTTATAAGTTGAACTCAAG-3' & This study \\
\hline ExbD2-F & 5'-AGTCGGATCCTTAGTGACTGGATTGCG-3' & This study \\
\hline ExbD2-R & 5'-AGTCCTGCAGTAACCTATTATTTGTCTAGT-3' & This study \\
\hline TonB-R & 5'-AGTCCTGCAGGCGATTTCTTCTTGGTG-3' & This study \\
\hline Orf2-R & 5'-AGTCCTGCAGATTAGAACTAATAGATTAG-3' & This study \\
\hline
\end{tabular}

${ }^{\star}$ Antibiotic resistance phenotypes: ampicillin, $\mathrm{Ap}^{\mathrm{r}}$; tetracycline, $\mathrm{Tc}^{\mathrm{r}}$. Antiobiotic resistance phenotypes and other features listed in parentheses are those expressed by F. psychrophilum but not by E. coli.

$\dagger$ Recognition sequences of BamHI (ExbD2-F) and PstI (ExbD2-R, TonB-R and Orf2-R) added to the sequence are underlined.

Siderophore quantification during growth of THC02-90 and FP1033 in NB was carried out using the Chrome Azurol S (CAS) assay as described by Schwyn \& Neilands (1987). At different incubation times $1 \mathrm{ml}$ aliquots were collected by centrifugation at $15000 \mathrm{~g}$ for $4 \mathrm{~min}$ and stored at $-20{ }^{\circ} \mathrm{C}$ until required. Siderophore units (\%) were defined as $\left[\left(A_{\mathrm{r}}-A_{\mathrm{s}}\right) / A_{\mathrm{r}}\right] \times 100$, according to Schwyn \& Neilands (1987).

$\mathbf{L D}_{\mathbf{5 0}}$ determination of FP1033. $\mathrm{LD}_{50}$ determinations were done using rainbow trout fry (Oncorhynchus mykiss; mean weight $5 \mathrm{~g}$ ), which were kept in $40 \mathrm{l}$ tanks containing dechlorinated water at $12 \pm 1{ }^{\circ} \mathrm{C}$ and acclimatized for a minimum of 3 days. F. psychrophilum cells from exponential-phase cultures of both THC02-90 and FP1033 were harvested by centrifugation and washed with PBS. Groups of 10 fish were challenged by intramuscular injection of $0.05 \mathrm{ml}$ of serial dilutions in PBS containing $10^{3}-10^{9}$ bacterial cells and $\mathrm{LD}_{50}$ was calculated according to the method of Reed \& Muench (1938).

Vaccination. The rainbow trout (mean weight $5 \mathrm{~g}$ ) were kept in the same conditions as described for the $\mathrm{LD}_{50}$ experiments. They were fed a commercial feed (Proaqua) at a rate of $1 \%$ biomass per day throughout the experiments. Vaccination trials were conducted on two 50-fish groups. Fish of the control group were injected intramuscularly with $0.05 \mathrm{ml} \mathrm{PBS}$, whereas fish of the other group were injected with $0.05 \mathrm{ml}$ PBS containing $10^{3} \mathrm{FP} 1033$ bacterial cells. After 6 weeks of immunization the fish were challenged with $1 \mathrm{LD}_{50}$ of the parental strain. The cumulative percentage mortality (CPM) was determined after 21 days, and the relative percentage survival (RPS) was calculated using the following equation: RPS $=[1-(\mathrm{CPM}$ of immunized trout/CPM of PBS-injected trout) $] \times 100$.

Genetic nomenclature and nucleotide sequence accession number. ORFs coding for proteins with amino acid sequences similar to proteins of known function were named after the corresponding genes. The remaining ORFs, which did not have high sequence similarity with known genes, were named orf2 and orf3.

The ton $B$ operon and surrounding DNA sequence has been assigned GenBank accession number EF408823.

\section{RESULTS AND DISCUSSION}

\section{Analysis of the Tn4351-disrupted sequence and the surrounding regions}

A set of $F$. psychrophilum mutants with defects in motility, proteolytic activity or the ability to grow in the presence of 2,2'-dipyridyl were previously obtained by Tn 4351 mutagenesis (Álvarez et al., 2006). Among them, strain FP1033, which lacked extracellular proteolytic activity on gelatin plates and grew poorly on iron-limited solid medium, was further 
analysed. Southern blot analysis revealed that FP1033 had a single Tn4351 insertion (data not shown). The DNA sequence surrounding the transposon insertion was obtained by inverse PCR as described by Álvarez et al. (2006). The disrupted sequence corresponds to an ORF of $543 \mathrm{nt}$ that codes for a protein of 180 aa and $19.8 \mathrm{kDa}$ (Fig. 1a). This protein exhibits sequence identity to predicted ExbD proteins from other micro-organisms such as Prosthecochloris vibrioformis (GenBank accession no. ZP_00661181; 32\%) and Chlorobium phaeobacteroides (GenBank accession no.

(a)

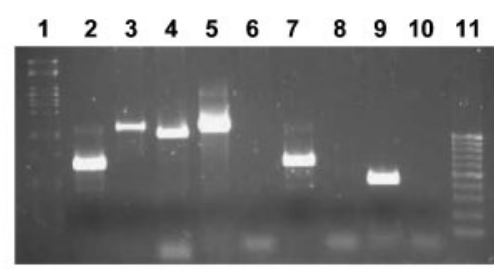

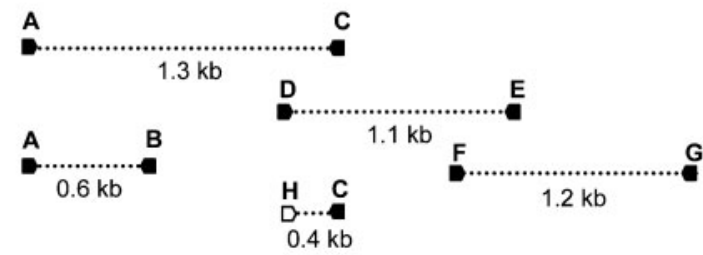

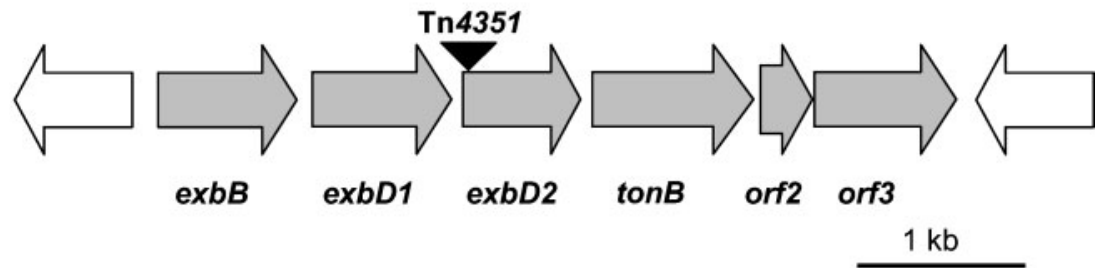

(b)

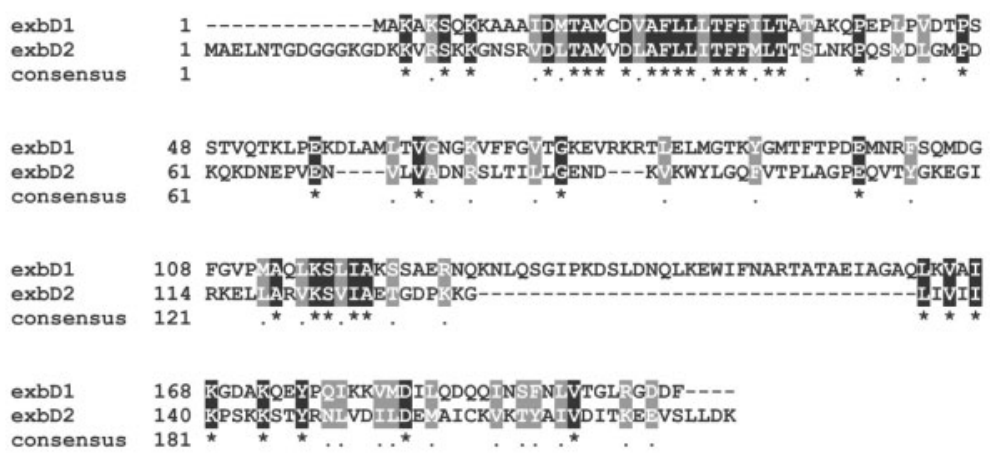

(c)

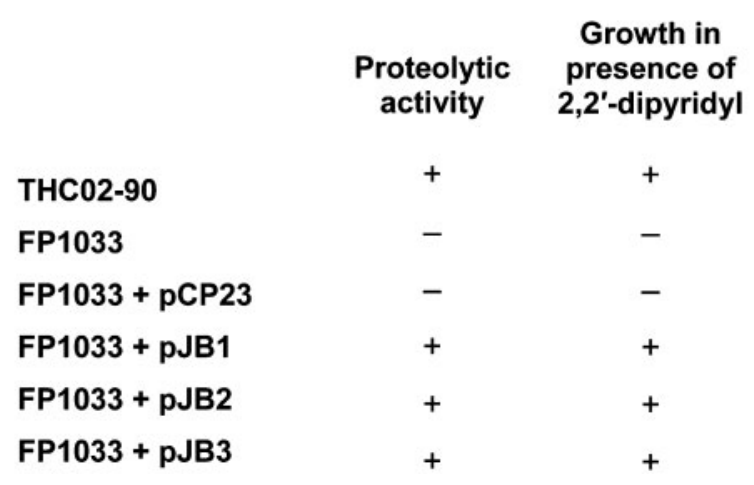

Fig. 1. (a) Organization of the genes, RT-PCR analysis and complementation studies in the ton $B$ cluster of $F$. psychrophilum. The position and orientation of genes are shown. The site of transposon insertion in the exbD2 locus is indicated. In the upper part of the schematic representation of the ton $B$ map, the position of each gene and the location of the primers used for RT-PCR amplifications are shown. The black arrows represent specific primers situated in operon genes (A, RT-exbB-F; B, RT-exbD1-R; C, RT-exbD2-R; D, RT-exbD2-F; E, RT-tonB-R; F, RT-tonB-F; G, RT-orf3-R) and the white arrow represents a specific primer situated in the transposon Tn4351 (H: RT-Tn). The size of the amplicons obtained is also indicated. The gel shows the products of RT-PCRs obtained from the parental (THC02-90) and the mutant (FP1033) strains. Lane 1, $\lambda$ DNA digested with Pstl as molecular marker; lane $11,100 \mathrm{bp}$ molecular marker; lanes 2-5, RT-PCRs performed with THC02-90 RNA; lanes 7-9, RTPCRs performed with FP1033 RNA. The following primers were used: $A$ and $B$ for lanes 2 and $7 ; A$ and $C$ for lanes 3 and $8 ; D$ and $E$ for lane 4; $F$ and $G$ for lane 5 ; and $H$ and $C$ for lane 9. Lanes 6 (parental strain) and 10 (mutant strain) are negative controls to determine whether RNA was free of contaminant DNA. (b) Alignment of F. psychrophilum ExbD1, ExbD2 and consensus ExbD, showing amino acid identities (black boxes; asterisk in consensus) and biochemically conservative substitutions (grey boxes; dot in consensus). The identity between ExbD1 and ExbD2 was $17 \%$ and similarity was $44 \%$. (c) Complementation of the exbD2::Tn4351 mutant. FP1033 cells containing plasmids pCP23 (cloning vector), pJB1 (exbD2), pJB2 (exbD2-tonB) and pJB3 (exbD2-tonB-orf2) were obtained and the indicated phenotypes analysed. 
ZP_00527754; $29 \%$ ). Nineteen nucleotides upstream of this exbD locus lies a locus that encodes a putative gene product with $28 \%$ sequence identity to a predicted ExbD/TolR protein from Prosthecochloris aestuarii (GenBank accession no. ZP_00591804). Based on these identities the loci were named exbD2 and exbD1, respectively. The deduced amino acid sequence of the exbD1 and exbD2 loci showed $17 \%$ identity and $44 \%$ similarity to each other (Fig. $1 b$ ). Additional nucleotide sequence analysis revealed three contiguous ORFs downstream of the exbD2 locus (Fig. 1a). The first ORF, with the start codon located $31 \mathrm{nt}$ downstream of the exbD2 stop codon, designated ton $B$, encodes a predicted protein of 272 aa that is $53 \%$ identical to the TonB-like protein from Flavobacterium johnsoniae (GenBank accession no. ZP_01247260). Downstream of tonB lies orf2, which encodes a hypothetical protein of 76 aa that does not exhibit sequence identity to proteins of known function. The orf 2 stop codon is followed by the orf 3 start codon. The amino acid sequence of the predicted orf 3 product of 301 aa was $54 \%$ similar to a putative $A B C$ transporter from $F$. johnsoniae (GenBank accession no. ZP_01247261). Finally, $65 \mathrm{nt}$ upstream of the exbD1 start codon is the stop codon of the exbB locus. The protein encoded by exbB is highly similar (78\%) to the MotA/TolQ/ ExbB proton channel from $F$. johnsoniae (GenBank accession no. ZP_01247257). Therefore, according to the identities, gene organization and the presence of characteristic elements of the TonB system, this cluster of genes is designated hereafter as the TonB system of $F$. psychrophilum (Fig. 1a).

An interesting finding was the presence of two contiguous similar exbD1 and exbD2 loci, located between the exbB and the ton $B$ genes. Composition of the TonB system is similar in most bacteria (TonB, ExbB and ExbD). To our knowledge, the existence in Gram-negative bacteria of two ExbD proteins encoded in the same cluster has only been reported for Xanthomonas campestris (Wiggerich \& Pühler, 2000). In this bacterium ExbD1 is involved in iron uptake whereas ExbD2 is not (Wiggerich \& Pühler, 2000). As for $X$. campestris, in F. psychrophilum the inactivation of the exbD2 gene demonstrated that it is involved in the iron uptake, probably by the TonB system. This more complex TonB system with an additional exbD locus could be related to an increase in its scope. The TonB system may be involved in the import of other compounds into the bacteria in addition to iron uptake (Postle, 1990; Braun, 1995; Köster, 2001). Further investigation is needed to determine the specific role of each of the two exbD genes in F. psychrophilum.

\section{RT-PCR analysis and complementation studies}

Transcription analysis of the loci $\operatorname{exb} B, \operatorname{exbD1}$, exbD2, tonB, orf 2 and orf 3 by RT-PCR in F. psychrophilum THC02-90 was carried out. Amplicons corresponding to overlapping transcripts from exbB to exbD1, exbB to exbD2, exbD2 to $\operatorname{ton} B$ and ton $B$ to orf 3 were obtained (Fig. 1a, lanes 2, 3, 4 and $5)$. These results confirmed that all these genes form part of an operon that was named the ton $B$ operon. In the case of
FP1033 no RT-PCR product was obtained from exbB to exbD2 (Fig. 1a, lane 8). However, an amplicon from FP1033 RNA was produced using a specific primer at the end of Tn4351 (RT-Tn) and the primer RT-exbD2-R, indicating that a promoter within the transposon drives the transcription of tonB, orf2 and orf3 (Fig. 1a, lane 9). Therefore, the insertion of the transposon in exbD2 caused a non-polar mutation and this result also demonstrates that orf 2 and orf 3 are indeed part of the tonB operon. As was previously indicated by experiments using Tn 4351 in Bacteroides fragilis (Smith et al., 1992) and F. johnsoniae (Hunnicutt \& McBride, 2000), our result confirms that Tn4351 possesses, at least at one end, an outwards-orientated promoter activity since DNA was generated when using primers situated inside Tn4351 and in the middle of the exbD2 gene.

Using the pCP23 expression vector (Álvarez et al., 2004, 2006), three plasmids, pJB1, pJB2 and pJB3, were constructed as described in Methods to complement FP1033. Introduction of $\mathrm{pJB1}$, which contained only exbD2, into FP1033 restored growth on iron-depleted medium (Fig. 1c). The same results were obtained with plasmids pJB2, which contained $\operatorname{exbD2}$ and ton $B$, and $\mathrm{pJB} 3$, which contained $\operatorname{exbD2}$, tonB and $f p o 2$. The complementation studies are in agreement with the RT-PCR results and indicate that the defects of FP1033 in growth on iron-depleted medium were caused by the lack of the ExbD2 protein.

\section{Phenotypic characterization of the FP1033 mutant}

In the presence of $50 \mu \mathrm{M}$ of the iron chelator 2,2' dipyridyl, growth of FP1033 in NB was inhibited, whereas a minor effect was found in the parental strain (data not shown). When the growth of the two strains was compared, it was clear that there is a significant difference between them in growth rate but not in the final yield (Fig. 2a). FP1033 showed a reduced growth rate in the exponential phase and a longer lag phase, delaying the growth by approximately $20 \mathrm{~h}$ in comparison with the parental strain (Fig. 2a). However, when the NB medium was supplemented with iron $\left(\mathrm{FeCl}_{3}\right)$ complete growth restoration was observed in the FP1033 strain (Fig. 2a).

Thus disruption of exbD2 resulted in inability to grow, or limited growth in iron-chelated or iron-deficient media, respectively. Nevertheless, growth of the mutant was not impaired in iron-sufficient conditions, since the growth rate was totally restored when an iron source was added to NB. These results indicate that the exbD2 mutation is physiologically irrelevant under the conditions tested in the presence of a sufficient amount of iron in the medium. We conclude that the role of the exbD2 gene seems to be related to scavenging iron in environments where this element is limiting.

Quantitative analysis of siderophores in the supernatants from the culture media throughout the growth curve from the parental and mutant strains showed that siderophore 

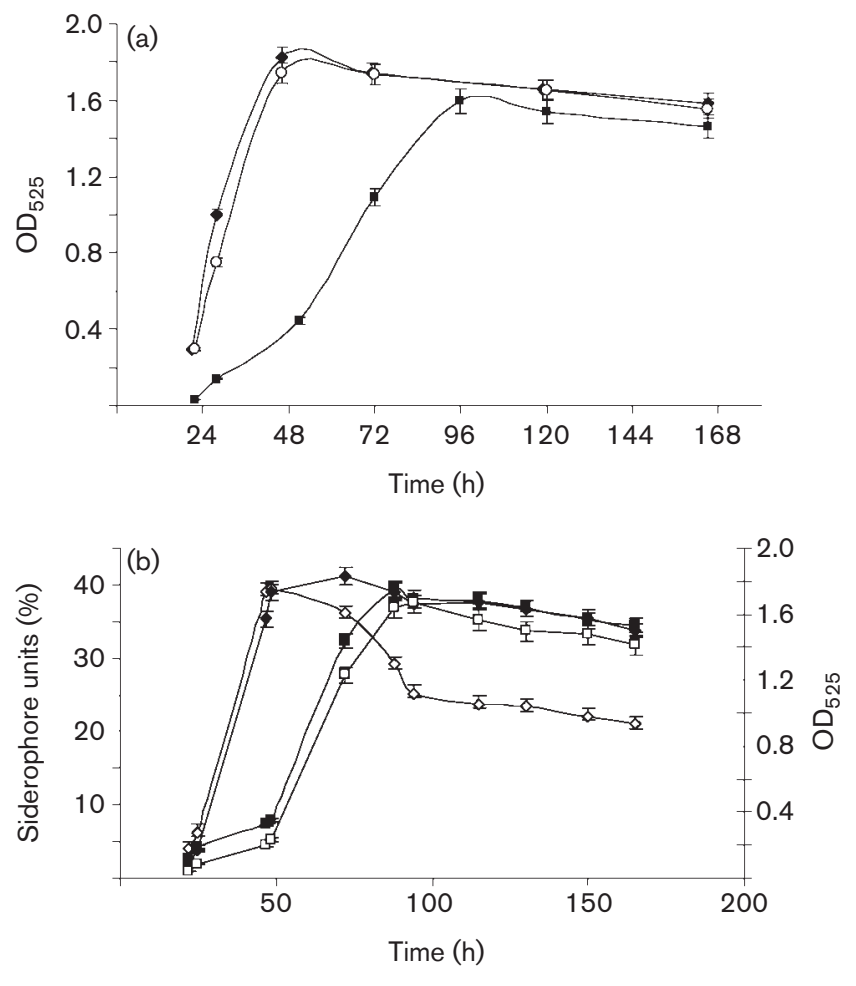

Fig. 2. (a) Growth curves of F. psychrophilum strains THC02-90 $(\boldsymbol{\nabla})$ and FP1033 ( $\boldsymbol{\square})$. The bacteria were grown in NB at $12{ }^{\circ} \mathrm{C}$. FP1033 was also grown in NB supplemented with $20 \mu \mathrm{M} \mathrm{FeCl}_{3}$ $(\bigcirc)$. Growth was monitored by determining the $O_{525}$. No effect on THC02-90 growth was observed in NB supplemented with iron. The growth curve of FP1033 complemented with pJB1 was similar to that shown for the parental strain. (b) Siderophore levels in the supernatant of the culture media of THC02-90 and FP1033 at different times of growth. $\boldsymbol{\nabla}$, Growth curves of THCO2-90 ( and FP1033 ( $\mathbf{\square}) . \diamond, \square$, Siderophore units, detemined as described by Schwyn \& Neilands (1987), in the supernatants of THC02-90 $(\diamond)$ and FP1033 $(\square)$. The results are means \pm SD $(n=3)$.

production was necessary for growth in NB and was growth stage dependent. In both strains the amount of siderophores increased during growth, reaching maximum values at the beginning of the stationary phase. However, a subsequent decrease in the level of siderophores occurred in the parental strain whereas no changes were found in the culture supernatant of FP1033 (Fig. 2b), suggesting that FP1033 has problems in the uptake of the iron-siderophore complex due to the interruption of the exbD2 gene, which generates a dysfunction in the TonB system.

FP1033 was also found to be defective in extracellular proteolytic activity. Up- and downregulation of extracellular enzymes by iron limitation has been described for different bacteria (Tokuda et al., 1998; Enard \& Expert, 2000; Maunsell et al., 2006). Although in some cases this regulation is part of a more complex pathway related to virulence (Beare et al., 2003), the function of this association remains unclear. F. psychrophilum is able to degrade haemoglobin (Nematollahi et al., 2003); thus the production of certain proteases related to haemolysis could perhaps be regulated by the levels of iron.

\section{$L_{50}$ determination and vaccination studies}

In order to elucidate the effect of the exbD2 mutation on the virulence of $F$. psychrophilum, $\mathrm{LD}_{50}$ determinations were carried out by infecting rainbow trout fry with the parental and the FP1033 strains. Ten days post-infection, $\mathrm{LD}_{50}$ values were $2.7 \times 10^{8}$ and $6 \times 10^{5}$, respectively. Thus, the mutant strain was approximately 450 -fold attenuated compared to the parent, indicating that inside the fish there is an iron-limited condition and a functional ExbD2 is required for full virulence in $F$. psychrophilum. The results obtained when in vivo competition assays between THC0290 and FP1033 cells were carried out were inconsistent due to the low and poorly reproducible recovery of both types of cells from fish. This is one of the problems of working with this fastidious bacterium.

The high level of attenuation of FP1033 indicates that it could be a good candidate for the development of a live vaccine. Therefore, a protection challenge was carried out in rainbow trout fry to determine whether FP1033 cells can induce an immune response against CWD. At 6 weeks post-vaccination with FP1033 the fish were injected with 1 $\mathrm{LD}_{50}$ of the parental strain. As can be observed in Fig. 3, 22 out of 50 fish in the non-vaccinated group fish died within 7 days, whereas only 4 out of 50 fish in the immunized group died. Taking these data, the RPS value was $81.8 \%$, indicating that FP1033 is capable of inducing high protection in rainbow trout fry against the disease. Moreover, after injecting the immunized fish with the parental strain, some of them developed a skin lesion that, in contrast to the fatal development of the lesion in the control fish, gradually healed. To our knowledge, this is the first time that an attenuated strain of F. psychrophilum has been used in vaccination experiments. In previous studies, the vaccines were based on fractions (Rahman et al., 2002; LaFrentz et al., 2004; Dumetz et al., 2006) or inactivated whole cells (Kondo et al., 2003; Madetoja et al., 2006). Although it is difficult to compare results from different vaccination studies due to the heterogeneity of the conditions used (fish species, fish weight and vaccine preparation), FP1033 cells conferred a similar level of protection to fish as other vaccines. It is also notable that, after the challenge, the control fish developed a skin lesion around the injection site prior to death, whereas only some of the vaccinated fish presented such a lesion, which cicatrized in a few days, indicating that an immune response had been generated. Live attenuated vaccines have been developed for other fish pathogens such as $V$. anguillarum (Norqvist et al., 1989), Aeromonas salmonicida (Vaughan et al., 1993), Aeromonas hydrophila (Hernanz Moral et al., 1998) and Y. ruckeri (Temprano et al., 2005). The use of an attenuated vaccine against $F$. psychrophilum may offer many advantages in comparison with classical 


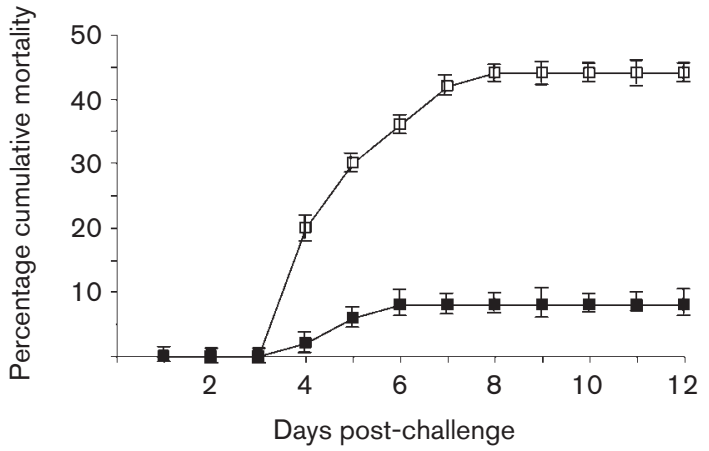

Fig. 3. Cumulative percentage mortality of rainbow trout fingerlings infected with THC02-90 with or without prior vaccination with FP1033. Fish of $5 \mathrm{~g}$ were intramuscularly injected with $10^{3}$ cells of FP1033, or PBS as control. Six weeks later fish were reinjected with $1 L_{50}$ of $\mathrm{THC02}-90$, and dead fish were quantified daily. $\square$, Control fish injected with PBS before reinjection with $\mathrm{THCO2-90;} \mathbf{\square}$, fish treated with $\mathrm{FP} 1033$ and then reinjected with THC02-90. The results are means $\pm \mathrm{SD}(n=3)$.

vaccine preparations, because the attenuated strain can survive in the host, stimulating its immune system without causing the disease. Thus, this vaccine may generate a prolonged immune response in fry which have an immature immune system. The results presented here establish the basis for the future development of a live attenuated vaccine that will be able to protect against CWD. Vaccine doses, bath immunization and cross-protection experiments using different strains are the next steps to carry out.

\section{ACKNOWLEDGEMENTS}

This work was supported by a grant from the MEC of Spain (AGL2006-07562). B. Á. and J.Á. were the recipients of grants from a SERIDA project and the MEC of Spain, respectively. We also thank J. Méndez for his continuous help and A. F. Braña for critical reading of the manuscript. We gratefully acknowledge Proaqua Nutrition SA for supporting this project.

\section{REFERENCES}

Agarwal, S., Hunnicutt, D. W. \& McBride, M. J. (1997). Cloning and characterization of the Flavobacterium johnsoniae (Cytophaga johnsonae) gliding motility gene, gldA. Proc Natl Acad Sci U S A 94, 12139-12144.

Álvarez, B., Secades, P., McBride, M. J. \& Guijarro, J. A. (2004). Development of genetic techniques for the psychrotrophic fish pathogen Flavobacterium psychrophilum. Appl Environ Microbiol 70, 581-587.

Álvarez, B., Secades, P., Prieto, M., McBride, M. J. \& Guijarro, J. A. (2006). A mutation in Flavobacterium psychrophilum tlpB inhibits gliding motility and induces biofilm formation. Appl Environ Microbiol 72, 4044-4053.

Andrews, S. C., Robinson, A. K. \& Rodriguez-Quiñones, F. (2003). Bacterial iron homeostasis. FEMS Microbiol Rev 27, 215-237.

Aoki, M., Kondo, M., Nakatsuka, Y., Kawai, K. \& Oshima, S.-I. (2007). Stationary phase culture supernatant containing membrane vesicles induced immunity to rainbow trout Oncorhynchus mykiis fry syndrome. Vaccine 25, 561-569.

Beare, P. A., For, J., Martin, L. W. \& Lamont, I. L. (2003). Siderophoremediated cell signalling in Pseudomonas aeruginosa: divergent pathways regulate virulence factor production and siderophore receptor synthesis. Mol Microbiol 47, 195-207.

Braun, V. (1995). Energy-coupled transport and signal transduction through the gram-negative outer membrane via ton $B$-exbB-exbDdependent receptor proteins. FEMS Microbiol Rev 16, 295-307.

Braun, V. \& Braun, M. (2002). Active transport of iron and siderophore antibiotics. Curr Opin Microbiol 5, 194-201.

Bruun, M. S., Schimdt, A. S., Madsen, L. \& Dalsgaard, I. (2000). Antimicrobial resistance patterns in Danish isolates of Flavobacterium psychrophilum. Aquaculture 187, 201-212.

Cabello, F. C. (2006). Heavy use of prophylactic antibiotics in aquaculture: a growing problem for human and animal health and for the environment. Environ Microbiol 8, 1137-1144.

del Cerro, A., Mendoza, M. C. \& Guijarro, J. A. (2002). Usefulness of a TaqMan-based polymerase chain reaction assay for the detection of the fish pathogen Flavobacterium psychrophilum. J Appl Microbiol 93, 149-156.

Dumetz, F., Duchaud, E., LaPatra, S. E., Le Marrec, C., Cleverol, S., Urdaci, M.-C. \& Le Hénaff, M. (2006). A protective immune response is generated in rainbow trout by an $\mathrm{OmpH}$-like surface antigen (P18) of Flavobacterium psychrophilum. Appl Environ Microbiol 72, 4845-4852.

Enard, C. \& Expert, D. (2000). Characterization of a tonB mutation in Erwinia chrysanthemi 3937: $\mathrm{TonB}_{E c h}$ is a member of the enterobacterial TonB family. Microbiology 146, 2051-2058.

Fernández, L., Márquez, I. \& Guijarro, J. A. (2004). Identification of specific in vivo-induced (ivi) genes in Yersinia ruckeri and analysis of ruckerbactin, a catecholate siderophore iron acquisition system. Appl Environ Microbiol 70, 5199-5207.

Garcia, C., Pozet, F. \& Michel, C. (2000). Standardization of experimental infection with Flavobacterium psychrophilum, the agent of rainbow trout Onchorhynchus mykiss fry syndrome. Dis Aquat Organ 42, 191-197.

Hernanz Moral, C., Flaño del Castillo, E., López Fierro, P., Villena Cortés, A., Anguita Castillo, J., Cascón Soriano, A., Sánchez Salazar, M., Razquín Peralta, B. \& Naharro Carrasco, G. (1998). Molecular characterization of the Aeromonas hydrophila aroA gene and potential use of an auxotrophic aroA mutant as a live attenuated vaccine. Infect Immun 66, 1813-1821.

Hunnicutt, D. W. \& McBride, M. J. (2000). Cloning and characterization of the Flavobacterium johnsoniae gliding-motility genes gldB and gldC. J Bacteriol 182, 911-918.

Izumi, S. \& Aranishi, F. (2004). Relationship between gyrA mutations and quinolone resistance in Flavobacterium psychrophilum isolates. Appl Environ Microbiol 70, 3968-3972.

Kondo, M., Kawai, K., Obake, M., Nakano, N. \& Oshima, S. (2003). Efficacy of oral vaccine against bacterial coldwater disease in ayu Plecoglossus altivelis. Dis Aquat Organ 55, 261-264.

Köster, W. (2001). ABC transporter-mediated uptake of iron, siderophores, heme and vitamin B12. Res Microbiol 152, 291-301.

LaFrentz, B. R., LaPatra, S. E., Jones, G. R. \& Cain, K. D. (2004). Protective immunity in rainbow trout Oncorhynchus mykiis following immunization with distinct molecular mass fractions isolated from Flavobacterium psychrophilum. Dis Aquat Organ 59, 17-26.

Madetoja, J., Lönnström, L.-G., Björkblom, C., Uluköy, G., Bylund, G., Syvertsen, C., Gravningen, K., Norderhus, E.-A. \& Wiklund, T. (2006). Efficacy of injection vaccines against Flavobacterium psychrophilum in rainbow trout, Oncorhynchus mykiis (Walbaum). J Fish Dis 29, 9-20. 
Maunsell, B., Adams, C. \& O'Gara, F. (2006). Complex regulation of AprA metalloprotease in Pseudomonas fluorescens M114: evidence for the involvement of iron, the ECF sigma factor, PbrA and pseudobactin M114 siderophore. Microbiology 152, 29-42.

McBride, M. J., Braun, T. F. \& Brust, J. L. (2003). Flavobacterium johnsoniae $\mathrm{GldH}$ is a lipoprotein that is required for gliding motility and chitin utilization. J Bacteriol 185, 6648-6657.

Michel, C., Antonio, D. \& Hedrick, R. P. (1999). Production of viable cultures of Flavobacterium psychrophilum approach and control. Res Microbiol 150, 351-358.

Møller, J. D., Ellis, A. E., Barnes, A. C. \& Dalsgaard, I. (2005). Iron acquisition mechanisms of Flavobacterium psychrophilum. J Fish Dis 28, 391-398.

Nematollahi, A., Decostere, A., Pasmas, F. \& Haesebrouck, F. (2003). Flavobacterium psychrophilum infections in salmonid fish. J Fish Dis 26, 563-574.

Norqvist, A., Hagström, A. \& Wolf-Watz, H. (1989). Protection of rainbow trout against vibriosis and furunculosis by the use of attenuated strains of Vibrio anguillarum. Appl Environ Microbiol 55, 1400-1405.

Payne, S. M. (1993). Iron acquisition in microbial pathogenesis. Trends Microbiol 1, 66-69.

Payne, S. M., Wyckoff, E. E., Murphy, E. R., Oglesby, A. G., Boulette, M. L. \& Davies, N. M. (2006). Iron and pathogenesis of Shigella: iron acquisition in intracellular environment. Biometals 19, 173-180.

Postle, K. (1990). TonB and gram-negative dilemma. Mol Microbiol 4, 2019-2025.

Pradel, E., Guiso, N., Menozzi, F. D. \& Locht, C. (2000). Bordetella pertussis TonB, a Bvg-independent virulence determinant. Infect Immun 68, 1919-1927.

Rahman, H., Kuroda, A., Dijkstra, J. M., Kiryu, I., Nakanishi, T. \& Ototake, M. (2002). The outer membrane fraction of Flavobacterium psychrophilum induces protective immunity in rainbow trout and ayu. Fish Shellfish Immunol 12, 169-179.

Reed, L. J. \& Muench, H. (1938). A simple method of estimating fifty percent endpoints. Am J Hyg 27, 493-497.

Reeves, S. A., Torres, A. G. \& Payne, S. M. (2000). TonB is required for intracellular growth and virulence of Shigella dysenteriae. Infect Immun 68, 6329-6336.

Schmidt, A. S., Bruun, M. S., Dalsgaard, I., Pedersen, K. \& Larsen, J. L. (2000). Occurrence of antimicrobial resistance in fish-pathogenic and environmental bacteria associated with four Danish rainbow trout farms. Appl Environ Microbiol 66, 4908-4915.
Schwyn, B. \& Neilands, J. B. (1987). Universal chemical assay for the detection and determination of siderophores. Anal Biochem 160, $47-56$.

Seliger, S. S., Mey, A. R., Valle, A. M. \& Payne, S. M. (2001). The two TonB systems of Vibrio cholerae: redundant and specific functions. Mol Microbiol 39, 801-812.

Simon, R., Priefer, U. \& Puhler, A. (1983). A broad host range mobilization system for in vivo genetic engineering: transposon mutagenesis in Gram-negative bacteria. Bio/technology 1, 784-791.

Smith, C. J., Rogers, M. B. \& McKee, M. L. (1992). Heterologous gene expression in Bacteroides fragilis. Plasmid 27, 141-154.

Stork, M., Di Lorenzo, M., Mouriño, S., Osorio, C. R., Lemos, M. L. \& Crosa, J. H. (2004). Two TonB systems function in iron transport in Vibrio anguillarum, but only one is essential for virulence. Infect Immun 72, 7326-7329.

Temprano, A., Riano, J., Yugueros, J., González, P., De Castro, L., Villena, A., Luengo, J. M. \& Naharro, G. (2005). Potential use of a Yersinia ruckeri $\mathrm{O} 1$ auxotrophic aroA mutant as a live attenuated vaccine. J Fish Dis 28, 419-427.

Tokuda, M., Chem, W., Karunakaran, T. \& Kuramitsu, H. K. (1998). Regulation of protease expression in Porphyromonas gingivalis. Infect Immun 66, 5232-5237.

Torres, A. G., Redford, P., Welch, R. A. \& Payne, S. M. (2001). TonBdependent systems of uropathogenic Escherichia coli: aerobactin and heme transport and TonB are required for virulence in the mouse. Infect Immun 69, 6179-6185.

Vaughan, L. M., Smith, P. R. \& Foster, T. J. (1993). An aromaticdependent mutant of the fish pathogen Aeromonas salmonicida is attenuated in fish and is effective as a live vaccine against the salmonid disease furunculosis. Infect Immun 61, 2172-2181.

Wandersman, C. \& Delepelaire, P. (2004). Bacterial iron sources: from siderophores to hemophores. Annu Rev Microbiol 58, 611-647.

Wiggerich, H.-G. \& Pühler, A. (2000). The exbd2 gene as well as the iron-uptake genes tonB, exbB and exbD1 of Xanthomonas campestris pv. campestris are essential for the induction of a hypersensitive response on pepper (Capsicum annuum). Microbiology 146, 1053-1060.

Wooldridge, K. G. \& Williams, P. H. (1993). Iron uptake mechanisms of pathogenic bacteria. FEMS Microbiol Rev 12, 325-348.

Wyckoff, E. E., May, A. R. \& Payne, S. M. (2007). Iron acquisition in Vibrio cholerae. Biometals 20, 405-416.

Edited by: S. C. Andrews 